\title{
Radiation Doses and Risks Associated with Mammographic Screening
}

\author{
Hilde Bosmans $\cdot$ Nicholas Marshall
}

Published online: 21 February 2013

(c) Springer Science+Business Media New York 2013

\begin{abstract}
Radiation dose and the associated risk to the breast are being studied in much detail with the aim of justifying breast cancer screening. The mean glandular dose has been proposed as the proper quantity to describe risks incurred by radiation. X. Wu (US) and D. Dance (UK) were the first authors to standardize breast dosimetry and their conversion tables have been used throughout. Doses can be calculated for specific population samples or for individual patients, in which case an accurate estimate of the glandular tissue fraction should be known. From specific DICOM tags in the digital images and access to tube output measurements from the medical physics QA, patient dose assessment can be largely automated. Other (traditional) approaches mimic clinical exposures on a Perspex phantom and use these exposures to estimate the breast dose to a typical breast. Any local data, both patient dose data and doses to Perspex, can these days be compared to an extensive amount of published dose values. Radiation dose and image quality should both be considered in screening applications. Better image quality can have a significant effect on diagnostic performance. As only a small increase in performance (ex. detected fraction) gained can mean an enormous amount of women saved, image quality settings should be optimized and not minimized. Risk benefit investigation should therefore focus on performance rather than dose. For a few screening programs, the benefits could be proven. Emerging technologies should be investigated along the same basic principles.
\end{abstract}

H. Bosmans $(\bowtie) \cdot$ N. Marshall

Medical Imaging Research Center (MIRC), University Hospitals of the KU Leuven, Herestraat 49, 3000 Leuven, Belgium

e-mail: hilde.bosmans@uzleuven.be
Keywords Breast cancer screening - Young women · Justification, glandular tissue - Mean glandular dose . Automated patient dosimetry $\cdot$ Diagnostic reference levels

\section{Introduction}

'Any proposed activity that may cause radiation exposure to persons should yield a sufficient benefit to society to justify the risks incurred by the radiation exposure.' This statement, known as the ALARA principle, is particularly valid in breast cancer screening. On that occasion, a specific portion of the population is invited for an X-ray examination. None of these women is a priori symptomatic for breast cancer.

Justification of X-ray mammography for screening applications examines the balance between the detriment induced by the examination and the benefit to the population. The first factor is based upon the dose to the breast and its associated risk. The second factor addresses the benefits of the screening program. A good indicator of benefit is the number of lives saved by the screening program, which takes into account the reduction in mortality that results from detecting cancer at an early stage when the prognosis is much better, the available treatment options and their success rate. An ultimate proof for the benefit of screening programs has to be found in downward trends of mortality. Often cancer detection rates and even more specifically small cancer detection ratios are used as a first parameter.

The risk and benefit of screening are under constant scrutiny $[1 \bullet \bullet]$. Today there are also worldwide debates triggered by cost-economic considerations. The debates are at international conferences as well as in a number of review papers. All of them take part in the decision processes regarding the roll-out and eventual practicalities of screening programs. Indeed, there are a number of 
decisions to be made, including the age range of invited women, the modality of screening, the number of views, the organization of second reading, the differentiation of women in terms of individual risk factors, etc.

Radiation protection sciences often express radiation detriment via the effective dose concept. This quantity multiplies the absorbed doses in different organs by an organ specific weighting factor. The weighting factor for the breast has been 0.05 in the International Commission on Radiological Protection (ICRP) report 62 and changed to 0.12 in the ICRP 103 report [2]. Effective dose has however limitations in the assessment of the risks from breast cancer screening, as the weighting factors represent an average value over men and women, over all ages or over the age of the 'working population'. The same ICRP documents as well as the reports of the Biological Effects of Ionizing Radiation of the National Academy of Sciences (BEIR VII) list age related detriments following absorbed doses to the breast and this approach is preferred.

Selected groups have addressed the risks and benefits associated with screening activities. Already in 1989, the Institute of Physical Sciences in Medicine (IPSM) recognized that the risks would be small but it is important that it be minimised, and an assessment of the dose to the breast should be included in any mammographic quality assurance programme [3]. Most of the radiation risk studies investigate at first the absorbed dose to the breast and estimate from there a number of radiation-induced cancers, with latency periods usually assumed to be at least ten years [3]. Given this long period, the fact that induced cancers cannot be distinguished from other cancers and the low numbers of induced cancers make their direct quantification today impossible.

In a review document in 2003 on radiation risk in breast screening [4•], the National Health Service Breast Screening Programme (NHSBSP) used radiation detriment induced risk factors as published by the Health Protection Agency (HPA), formerly the National Radiological Protection Board (NRPB). This allowed age specific risk assessment. The following conclusions were reached for the screening procedures in the UK, assuming a dose per breast of $4.5 \mathrm{mGy}$ for a 2 view acquisition:

- the risk of a radiation-induced cancer for a woman attending mammographic screening (two views) by the NHSBSP is about 1 in 20,000 per visit

- it is estimated that about 170 cancers are detected by the NHSBSP for every cancer induced

- the mortality benefit of screening exceeds the radiationinduced detriment by about 100:1

- for the very small proportion of women who receive the highest radiation doses, the benefit will exceed the risk by about $20: 1$.
In 2010, Hendrick [ $\left.1{ }^{\bullet \bullet}\right]$ based their analysis upon the sex and 'age at exposure' specific risk factors as found in BEIR VII. The age specific risk factors allowed risk assessment for specific screening settings, such as 'younger women', elder women, yearly and 2 yearly programs. Their results are favorable for actual breast cancer screening programs: the life attributable risk of radiation induced breast cancer is estimated to be 31 per 100,000 women screened with an absorbed dose to the breast of $3.7 \mathrm{mGy}$ and mortality of 10 per 100,000 women screened in an annual screening regime. Yaffe and Mainprize [5•*] concluded in another study that for screening programs that begin at age 40 years, the risk of radiation-induced cancer is small compared with the expected mortality reduction achievable through screening. The risk of radiation-induced breast cancer should not be a deterrent from mammographic screening of women over the age of 40 years. They used an absolute risk model instead of a relative risk model. Their conclusions hold even when taking the uncertainties on risk factors into account. To test this, they reduced the latency to 0 years, and considered the survival rates to be those of an unscreened population. Some authors focus specifically on the younger women, with ages in the range $40-50$ years [6].

It is clear from a review of current literature that the mean glandular dose acts as a scaling factor in the risk benefit analysis of the breast cancer screening programs. This underlines the role of breast dose assessment.

\section{Recent Evolutions in Breast Cancer Imaging and its Dosimetry}

Breast imaging is going through an exciting period with many new modalities or techniques brought onto the market. Today, there is a rapid transition from film-screen mammography to digital mammography. Different digital technologies can be considered, including computed radiography (CR) for mammographic applications with powder or needle based photostimulable phosphor plates, and direct radiology (DR) using properties of scintillation, amorphous selenium or photon counting. The dose levels at which these systems are operated should be adopted to the image quality that can be obtained with these systems as the performance is different among the different systems $[7,8 \bullet \bullet, 9 \bullet \bullet$.

With regard to emerging new candidate modalities for breast cancer screening, we can distinguish between technologies providing 3D information (e.g.,: breast tomosynthesis and breast CT), perfusion related parameters (e.g.,: dual energy subtraction methods), spectral data without contrast agents (e.g.,: using photon counting detectors), and other physics parameters (e.g.,: phase contrast imaging). 
Their eventual widespread application (in screening) requires a specific or generic justification first. As far as we know today, the same components will define this process: dose to the breast (risk) and effects on mortality reduction (benefit). Their advantages may come from better cancer detection or from other indicators such as better positive predictive value for differentiation between benign and malignant lesions and less required add-on modalities. Advantages could also come from a more cost effective approach when recall rates are reduced or more expensive procedures can be avoided. A rapid introduction of justified emerging techniques may lead to a more pronounced impact of screening activities on breast cancer mortality.

The doses to the breast are linked to the X-ray modality and how it is being used. Thierry-Chef et al. [10] started their dose review study in 1960 and described a surprisingly large spread in doses of a factor of 100 on the early $\mathrm{X}$-ray mammography systems, compared to about 5 these days. Huda et al. [11] reported the absorbed doses to the breast during screening in the time period 1978-1988. In the late 1980s, the AGD due to xeroradiography for a craniocaudal view was $3.3 \mathrm{mGy}$, a factor of 2.4 greater than the corresponding dose associated with dedicated screen-film units. Young et al. [12•] found that a typical woman in the UK got a dose of $4.5 \mathrm{mGy}$ for a 2 view filmscreen exam in 2001-2002.

Today, the NHS publishes comparative dose studies for both film-screen and all digital modalities on their website (http://www.cancerscreening.nhs.uk/breastscreen/publications/ mammography-equipment.html). A complicating factor with digital mammography is that systems can be used with a wide range of dose settings: with film-screen mammography, the optical density largely fixed the detector air kerma. In digital imaging, this condition does not apply anymore. In practice, three groups of people decide about the dose: the radiologist as they are responsible for quality in general, the manufacturer who proposes default/operational settings and the medical physics expert who has to optimize X-ray exposure settings and approves the settings being used. In 2007, Svahn et al. [13] calculated the number of deaths prevented as a result of screening mammography for the Malmö region, and the estimated overall effects following a dose reduction of $50 \%$. The study supports the benefit from dose reduction possibly achievable with new digital equipment. They estimate the number of prevented deaths as 200 per 100,000 women screened annually in the age group 40-49 years. The number of fewer breast cancer mortalities due to a dose reduction of $50 \%$ is expected to be 5.2 (using the NRPB model).

At the introduction of a new technique, absorbed doses are often communicated, sometimes in relation to other modalities like classical 2 view mammography. Boone, at the introduction of breast-CT, said that a simple scan would result in the same dose as 2 view mammography [14]. Kalender et al. [15] announced a mean glandular dose for breast CT of $3 \mathrm{mGy}$.

Contrast enhanced mammography using a high energy and low energy acquisition involves more than 1 standard planar exposure, yet companies try to stay with the dose equivalent to a standard planar X-ray exposure. Schmitzberger et al. [16] calculated an absorbed dose of only $3 \times 0.42 \mathrm{mGy}$ using the sectra microdose system, or $1.26 \mathrm{mGy}$ per breast in total. Dual energy digital breast tomosynthesis has been shown to be feasible too [17].

For all the above reasons, breast dosimetry remains a topic of current interest. Dose surveys are more mandatory now than in the film-screen period due to wider exposure ranges that can be selected. It is also important to achieve an international consensus on how to calculate breast dose for the newest modalities and to investigate how the doses of these new modalities compare with current techniques.

\section{Mean Glandular Dose}

X-ray mammography uses an X-ray beam approximately perpendicular to the compressed breast. The X-ray energy is selected by the user or is fully automatically determined by the automatic exposure device of the modality. The same device also regulates the quantity of delivered X-rays. The incident radiation beam determines the dose to the breast. For X-ray dosimetry purposes, the glandular tissue is of importance as X-ray induced malignant processes are thought to originate in the gland only. The glandular tissue is a part of the breast, with a total amount and spatial distribution of the tissue that is quite variable among women. Accurate dosimetry would require detailed information on both aspects. Whereas the total amount of glandular tissue and its distribution in an X-ray projection image can be quantified these days from mammograms and has shown to be very different from a mixture with $50 \%$ adipose and $50 \%$ glandular tissue [18••], the distribution along the third dimension remains unknown with current planar projection imaging techniques.

Notwithstanding the intrinsic uncertainty on the glandular distribution, the mean dose to the glandular tissue (MGD) has been proposed as the determining factor for detriment calculations. Conversion factors have been proposed to relate incident radiation to the MGD. They are based upon models of the breast, and more in particular, the shape of the breast, the thickness of the compressed breast and the amount and distribution of the glandular tissue. A number of authors have produced tables with conversion factors for different beam qualities, that are differentiated via anode/filter combination, tube voltage and the half 
value layer, as well as breast thickness and glandularity. Correction factors for other views (cranio-caudal views versus oblique views ...) are negligible. Workers in the USA would typically use the work of Wu et al. [19] of 1991, whereas Europeans tend to use D. Dance's factorial approach first proposed in 1990 [20••]. Both started from a homogeneous 50-50 distribution of adipose and glandular tissue. In a recent study, Sechopoulos et al. [21•] found that the conversion factors from incident air kerma to mean glandular dose were, on average, $27 \%$ higher using the classical model of a breast that is a homogenous mixture of adipose and glandular tissue than a model using a true heterogeneous glandular tissue distribution.

Sechopoulos et al. [22] and Dance et al. [23••] have also extended their tables to include tomosynthesis dosimetry too. Thacker and Glick [24] calculated the factors for flat panel breast CT.

Given the influence of the fraction of glandular tissue on the dose to the gland, population averaged values of glandular fraction have been obtained in the UK for screened women in the age range 50-64 years, and also for the so-called young women, with ages between 40 and 50 years. A result is shown in Fig. 1 [25].

Individualized patient dosimetry requires a patient specific density assessment. Radiologists can be asked to score the glandularity of the mammograms, via a categorization into typically four categories, based upon Wolfe's parenchymal pattern and percentage of the breast proposal. This approach is known to be highly subjective. Objective, quantitative methods to determine the glandular fraction can be summarized as follows: test slabs simulating different glandular tissue fractions are irradiated for set amounts of radiation output (fixed tube load in terms of $\mathrm{mAs}$ ) and beam quality and the resulting pixel values are

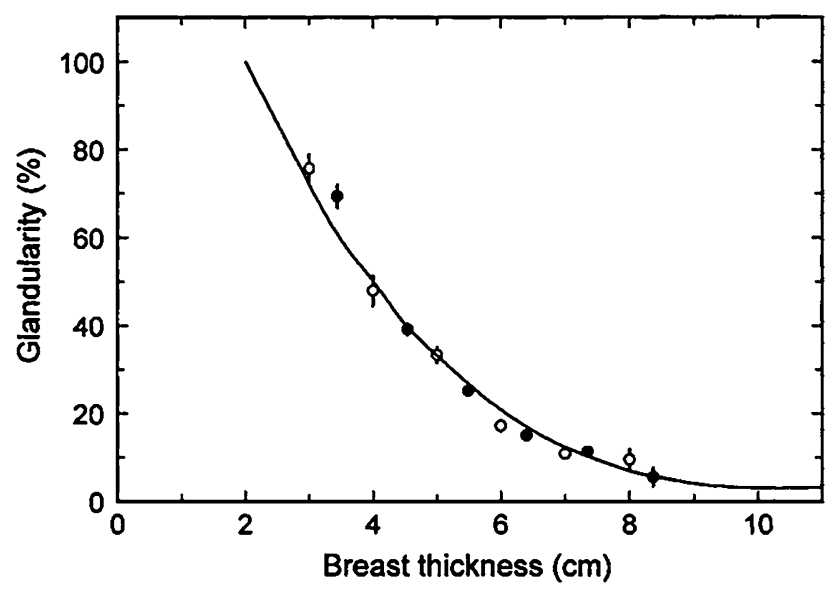

Fig. 1 Estimates of average breast composition for different compressed breast thickness in Guildford (open circles) and the Northern Region (full circles), for women in the age range 50-64 years. From [25], with permission registered. Parametric equations relating pixel values in the image and glandularity are then derived and can be adjusted towards any tube load setting. The inverted equation (or similar approaches) can in theory be applied to all pixel values in an image for any tube load to retrieve the glandular fraction in every point [26]. A similar method had been established before also for film screen systems [27]. The GE Digital mammography systems calculate an average glandular fraction in a region of the image and note the value in a DICOM tag. An analysis from a very large and international data sample showed the distribution of this glandular fraction as a function of the breast thicknesses [28]. The curve relating glandularity and compressed thickness, as obtained earlier for the UK [25], is confirmed in this analysis (Fig. 2). The study showed also differences between the US, Europe and Asia. Recently, Kotre could derive a link between glandularity and cancer detection in a cohort of women [29••].

\section{Patient Dose Surveys in Mammography}

In comparison to other organ dose applications in radiology, breast dosimetry is well established. With a limited set of input parameters, the mean glandular dose can be calculated. The most traditional approaches use the conversion factors of Wu et al. or Dance et al. Most of the input parameters are available in the mammograms: the anode/ filter combination, the tube load (mAs) and the compressed breast thickness. Parameters such as the half value layer and the tube output are available in the test reports of the medical physicist. A dose analysis can be further differentiated towards the mammographic view, the use of large or small compression paddles and magnification views.

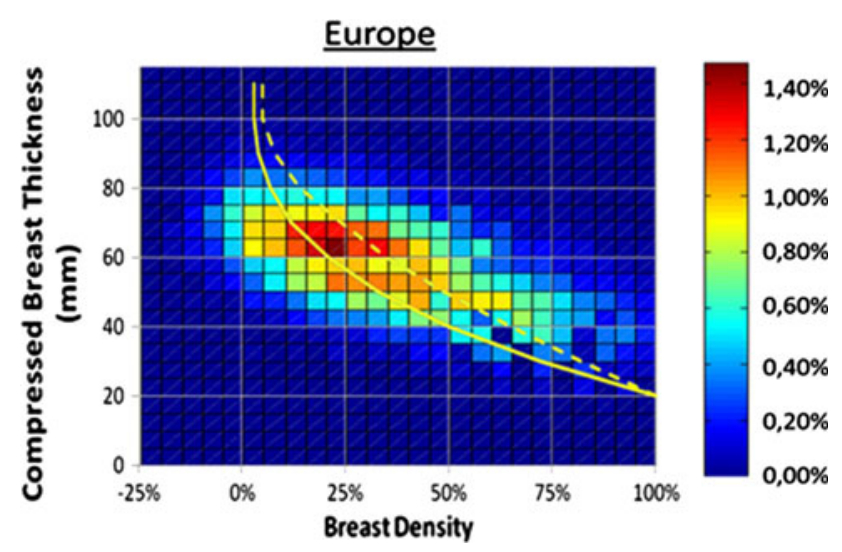

Fig. 2 Compressed breast thickness as a function of breast density calculated for a large patient sample; images acquired on systems of GE Healthcare (Buc, France) and peak breast density retrieved from the DICOM header of the images (lines: Data from Dance et al. [25], full line 50-64 years, dotted line 40-49 years). From [28], courtesy of the IAEA 
The glandular fractions of individual women can be used or population averaged values can be applied, which is the more common approach. Most scientists restrict themselves to 2 view mammography, they do not differentiate between the cranio caudal views versus the oblique views and they use the averaged glandular fractions of the UK screening population (Fig. 1). With all input parameters available, mean breast dose is obtained from selected searches in tables and multiplication of a series of factors.

Already in 2001, Rosenberg et al. [30] described how to automate data collection and analysis. In digital imaging, this process can be automated if the mammogram specific parameters are available in the DICOM header. Chevalier et al. applied this approach for a large patient dose survey on their digital mammography system [31••] and were the first to publish data of a large data sample imaged on a single system. Such a technique can be applied on most DR systems but is often not possible for $\mathrm{CR}$ as the required DICOM header information is usually not available. The best dose survey is continuous and on-line.

In Fig. 3, we show the results of a typical patient sample imaged with a digital mammography system and with breast tomosynthesis (both modalities from Siemens NV, Germany) [32]. The large sample is summarized in a curve that shows average doses as a function of compressed breast thickness. Data acquisition and dosimetry were automated (own data; software by qaelum NV, Belgium). Achievable and acceptable levels, that are technical measures, are plotted on top of the patient data.

\section{Predicting Patient Dose from the Standard Breast Phantom}

Phantom measurements may be more accessible than patient dose data. This is especially the case if the phantom acquisition can be incorporated in the test protocol of the medical physics expert. Hence, one of the objectives of the medical physics test is to ensure the safe use of the equipment and predicting patient dose, or at least be able to verify whether a system would generate an acceptable dose under standard conditions. In mammography, there is a long tradition with the use of such test objects. In the UK, a PMMA phantom of $4 \mathrm{~cm}$ thickness has been proposed as representative for breasts with glandularity of $50 \%$ and a compressed breast thickness of $4.5 \mathrm{~cm}$ [33••]. The European Guidelines proposed a $4.5 \mathrm{~cm}$ PMMA test block [48•]. The Mammography Quality Standards Act (MQSA, US) uses $4.2 \mathrm{~cm}$ of Perspex as the standard breast. Dance et al. [34] have recently re-evaluated the equivalence between Perspex blocks and compressed breast thickness for a large range of beam quality selections. Next to the use of computer simulations, the breast equivalent PMMA thickness can also follow from practical measurements that find the PMMA thickness that induces the same automatic exposure values as a group of patients with a typical breast thickness. This study was recently performed for the Taiwanese population [35].

The PMMA blocks are simple test objects, they are inexpensive and the risk for errors is minimal. It is important though to apply a typical compression force and to use the routine clinical protocol. Faulkner et al. [33••] showed a correlation between breast dose and dose to PMMA blocks, justifying the use of the test block. In that period, film-screen and mainly Molybdenum/Molybdenum anode/filter combinations were used with a tube voltage of $28 \mathrm{kV}$. If results have to be compared between centers and if the test block acts as an alternative for patient dosimetry, it is important to verify the thickness of the test blocks. In quality assurance (QA) protocols, limiting values on dose to such phantoms have been tabulated. In [36], it was shown that centres violating the dose limits on test blocks would also have high patient doses. It is important at this point to stress that dose limits to PMMA blocks should not be used to stop exposures to individual patients. In patients with dense breasts, the mean glandular dose can eventually be higher than routinely applied dose limits for the standard test block.

\section{Diagnostic Reference Levels in Mammography and Large Scale Dose Data Samples}

Patient dose surveys are considered an important tool in the optimization of X-ray imaging and of help in avoiding unnecessarily high doses to the patient. The EC published Guidelines on the collection of 'diagnostic reference levels' (DRLs) in 1999 [37]. They defined 'DRLs' as 'dose levels in medical diagnostic practices for typical examinations for groups of standard-sized patients or standard phantoms for broadly defined types of equipment. These levels are expected not to be exceeded for standard procedures when good and normal practice regarding diagnostic and technical performance is applied'. Once DRLs are available, new patient dose surveys can be compared to this reference. Especially if a large variation of dose is seen in a country, it may be advisable to start the investigation of a national DRL. This was the case at the introduction of digital mammography in Greece [38•].

The EC document states that for mammography, a standard phantom should be used. The use of phantoms as an alternative to patient dose surveys was in the nineties routine practice in the UK [33••]. This approach was later completed with large scale patient dose studies [12•]. Many countries have studied their patient doses too now, and established local DRLs [36, 39-42]. This happened 
Fig. 3 Comparison between doses from 2D mammography (2D MGD) (blue dots) and breast tomosynthesis (3D MGD) doses (red squares) as a function of breast thickness (Color figure online)

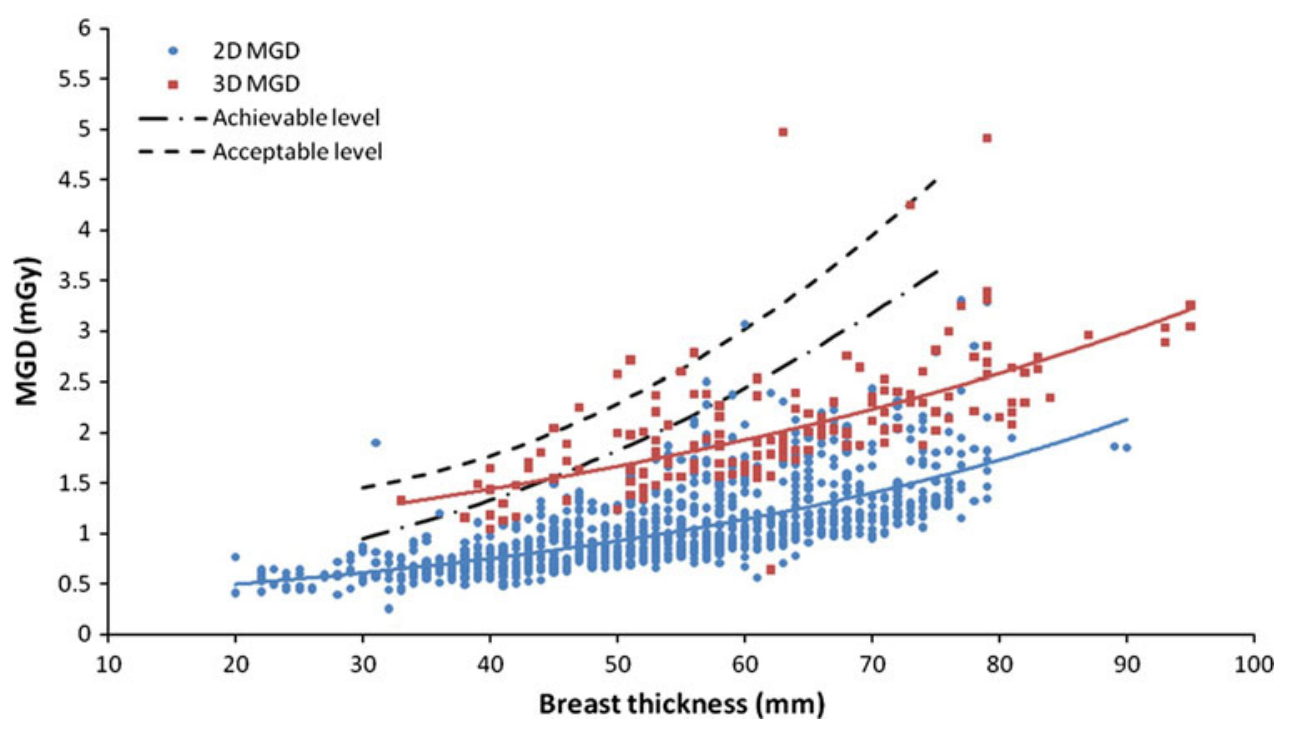

especially in the frame of screening programmes. Other member states started from the European DRL of $10 \mathrm{mGy}$ incident exposure.

Geeraert et al. [28] collected patient exposure data as available in a worldwide set of digital mammography systems and calculated dose distribution for Europe, the US and Asia. The results are summarized in Table 1.

The American breasts are largest and the Asian breasts are densest. Mean doses were for Europe, the US and Asia: $1.48,1.42$ and $1.42 \mathrm{mGy}$. This way of patient dose data supervision is unique: it underlines the fact that patient dosimetry can be automated if proper dose related data are saved with the images or saved in particular files in the system. Most digital systems indeed estimate MGD and store this information also in the DICOM header. McCullagh et al. [39] showed in an Irish study that system calculated MGD and offline calculated values can be reasonably close. It is recommendable that system available values are verified prior to their use. Informaticians can provide us with the tools to retrieve all the input data for subsequent organ dose calculations in an independent way. On-line dosimetry in digital (DR) mammography, showing how the dose of a patient relates to the dose distribution of a particular system, can become common practice too: all input for eventual software applications are available in the images. A more

Table 1 Statistics for the MGD distributions computed from the Senographe DS and Essential (GE HealthCare, Buc, France)

\begin{tabular}{llllll}
\hline & $\begin{array}{l}\text { Average } \\
(\mathrm{mGy})\end{array}$ & $\begin{array}{l}\text { Median } \\
(\mathrm{mGy})\end{array}$ & $\begin{array}{l}<25 \% \\
(\mathrm{mGy})\end{array}$ & $\begin{array}{l}<75 \% \\
(\mathrm{mGy})\end{array}$ & $\begin{array}{l}<95 \% \\
(\mathrm{mGy})\end{array}$ \\
\hline Europe & 1.48 & 1.4 & 1.2 & 1.6 & 2.4 \\
North-America & 1.42 & 1.4 & 1.2 & 1.6 & 2.1 \\
Asia-Pacific & 1.42 & 1.3 & 1.1 & 1.7 & 2.3 \\
\hline
\end{tabular}

From [28], courtesy of the IAEA complete picture of the radiation induced detriment in breast cancer screening programmes evaluates the number of mammograms that women get during the whole programme and the mean number of add-on X-ray imaging along with its MGDs. Faulkner et al. [34••] found the contribution of the MGD due to the mammograms to be dominant.

\section{The Often Forgotten Dose Related Factor: Diagnostic Image Quality}

Medical exposures are different from other sources of manmade radiation: it is assumed that the use of X-rays improves the quality of life in the population. Justification of breast cancer screening evaluates the ultimate benefit of these exposures and is one of the best studied ALARA questions in medicine. Some screening programmes could prove that a mortality reduction can be obtained. This is so far only proven for film-screen systems and it is assumed that digital modalities would obtain the same mortality reduction if diagnostic performance is not worse. Numerous studies have, therefore, compared image quality figures of filmscreen and the newer digital mammography systems.

It is a challenge to compare diagnostic performance between different modalities. Two scientific problems have to be solved: (1) what is a clinically relevant performance test for a given modality, and (2) how to compare between modalities.

One approach focuses on the most critical aspects of performance. An example is the detectability of small clusters of microcalcifications. As detectability is related with the contrast generated between the object to be detected and the background tissue, Figure of Merits (FoMs) have been defined in terms of contrast to noise tests or contrast detail analyses. Simulation experiments as well as practical measurements have been performed recently to 
understand and optimize digital mammography systems. Results show decreasing contrast to noise ratio or contrast detail scores at lower doses. Some studies aim to find optimal working points within a given range [44-47]. The European Guidelines on Quality Assurance in Breast Imaging [48•] have put absolute limits for contrast detail scores based on typical results obtained with film-screen systems in established screening programmes. The IAEA protocol for QA in breast cancer screening [49] bases acceptability criteria upon signal difference to noise ratios. As the absolute value of signal difference to noise ratio has no meaning, this measure cannot be used across systems and the QA protocol of the IAEA provides therefore a table with system specific limiting values. Klausz and Shramchenko [50] introduced an interesting new concept: the design for the automatic exposure controller of a digital mammography system can aim for an optimal dose to the population, computed by averaging the MGD values over the distribution of breast types in the population.

A second approach to quantify the quality in mammography uses 'mathematical observers'. Results of such an investigation are detectability indexes d'. Different degrees of sophistication are possible: mathematical objects versus irregular shapes, homogenous backgrounds versus clustered lumpy backgrounds and even patient images, the choice of eye filter, etc. In selected studies, a correlation between the results of mathematical observers and the more simple contrast detail data could be shown [51]. Detectability d' can be used across systems.

Emerging mammography systems are being tested with similar strategies [52-55]. Another approach aims to include the complete imaging chain into the analysis [56].

The studies mentioned above are usually performed on raw data. They study the intrinsic contrast generated by selected objects that are representative for small high contrast objects or larger low contrast tumoral masses. The unique properties of digital imaging via software applications remained untested. In the film-screen era, the shape of the sensitometric curve was very well investigated as items such as a high gradient were known to enhance the visibility of some mammographic features. Software applications may have a similar effect. Their full capacity can only be tested on mammograms. This is an argument in favor of clinical trials. There are however also faster and less expensive approaches by using simulation. The complete imaging system could be simulated, from the X-ray tube and beam quality selection, a computer model of the breast with particular details of interest, the simulation of X-ray absorption by the detector followed by image processing and observer performance of the simulated details. This approach is known as the 'virtual clinical trial'. This has not yet been fully worked out. Partial experiments can be considered as preparatory steps for the larger aim of the complete simulation. Partial simulation can start from the raw data of real anatomical backgrounds and simulate details into these raw data. These hybrid images can then be processed and detail detectability can be assessed. Raw data could also be manipulated first to represent lower dose images or images acquired with a different detector. One study showed a definitive effect of processing on detectability of clusters of microcalcifications [57] whereas another study investigating other types of processing could not detect a difference [9*0]. There were remarkably large differences as a function of dose and for a DR system versus simulated CR systems [9・•]. These preliminary results suggest that it would be worth testing many more system configurations. A lot of research opportunities remain with complete system testing of emerging technology and in particularly also with the set-up of comparative tests between more established techniques and the new developments.

\section{Conclusion}

Risks and benefits of breast cancer screening programmes have been studied in much detail. The mean glandular dose is the quantity being used in these studies to express the risks. With emerging new technologies, that are candidates for use in screening programmes, more justification efforts will be needed. It is important to go beyond ratios such as 'detected cancers to cancers induced', as the advantages of new technologies may optimize other factors that ultimately lead to a larger mortality reduction.

Acknowledgments The authors want to thank their co-workers for the enthusiasm with which they work towards optimal breast cancer screening, both in the frame of Research Projects as well as during routine QA tasks.

Disclosure No potential conflict of interest relevant to this article was reported.

\section{References}

Papers of particular interest, published recently, have been highlighted as:

- Of importance

•- Of major importance

1. •- Hendrick RE. Radiation doses and cancer risks from breast imaging studies. Radiology. 2010;257(1):246-53. Special report paper that compares the risks associated with $X$-ray acquisitions of the breast and nuclear medicine exams and concludes that the life attributable risks of the latter exams are significantly higher than in classical 2D mammography.

2. The 2007 recommendations of the International Commission on Radiological Protection. International Commission on Radiological Protection. ICRP publication 103. AnnICRP. 2007;37(2-4): $1-332$. 
3. NHS report 54. Review of radiation risk in breast screening. Feb 2003.

4. - Risks to the UK Population. Document of the NRPB, vol. 4, no. 4. Chilton, NRPB, 1993. Historical text that set risk assessment guidelines.

5. • Yaffe MJ, Mainprize JG. Risk of radiation-induced breast cancer from mammographic screening. Radiology. 2011;258(1): 98-105. Recent paper that guides the reader through the process of estimating the risks of radiation induced cancers for various actual screening scenarios.

6. Feig SA, Hendrick RE. Radiation risk from screening mammography of women aged 40-49 years. J Natl Cancer Inst Monogr. 1997;22:119-24.

7. Lazzari B, Belli G, Gori C, Rosselli Del Turco M. Physical characteristics of five clinical systems for digital mammography. Med Phys. 2007;34(7):2730-43.

8. - Marshall NW, Lemmens K, Bosmans H. Physical evaluation of a needle photostimulable phosphor based CR mammography system. Med Phys. 2012;39(2):811-24. Strictly comparative technical study of most currently available systems on the market. The study confirms the differences between systems, and in particular also between $C R$ and DR technology.

9. •- Warren LM, Mackenzie A, Cooke J, et al. Effect of image quality on calcification detection in digital mammography. Med Phys. 2012;39(6):3202-13. Important paper showing that dose setting and detector technology may have an impact on the detection rate of clusters of subtle microcalcifications. The consequence is that it may be needed to adjust the levels of 'acceptable quality'.

10. Thierry-Chef I, Simon SL, Weinstock RM, et al. Reconstruction of absorbed doses to fibroglandular tissue of the breast of women undergoing mammography (1960 to the present). Radiat Res. 2012;177(1):92-108. Epub 2011 Oct 12.

11. Huda W, Sourkes AM, Bews JA, Kowaluk R. Radiation doses due to breast imaging in Manitoba: 1978-1988. Radiology. 1990;177(3):813-6.

12. - Young KC, Burch MA, Oduko JM. Radiation doses received in the UK Breast Screening Programme in 2001 and. Br J Radiol. 2002;78(2005):207-18. First large scale dose assessment study in breast cancer screening.

13. Svahn T, Hemdal B, Ruschin M, et al. Dose reduction and its influence on diagnostic accuracy and radiation risk in digital mammography: an observer performance study using an anthropomorphic breast phantom. Br J Radiol. 2007;80:557-62.

14. Boone JM, Kwan AL, Yang K, et al. Computed tomography for imaging the breast. J Mammary Gland Biol Neoplasia. 2006; 11(2):103-11

15. Kalender WA, Beister M, Boone JM, et al. High-resolution spiral $\mathrm{CT}$ of the breast at very low dose: concept and feasibility considerations. Eur Radiol. 2012;22(1):1-8. Epub 2011 Jun 9.

16. Schmitzberger FF, Fallenberg EM, Lawaczeck R, et al. Development of low-dose photon-counting contrast-enhanced tomosynthesis with spectral imaging. Radiology. 2011;259(2):558-64.

17. Carton AK, Gavenonis SC, Currivan JA, et al. Dual-energy contrast-enhanced digital breast tomosynthesis-a feasibility study. Br J Radiol. 2010;83(988):344-50. doi:10.1259/bjr/ 80279516.

18. $\bullet$ Yaffe MJ, Boone JM, Packard N, et al. The myth of the 50-50 breast. Med Phys. 2009;36(12):5437-43. It is often assumed that the breast is a 50-50 mixture of glandular and adipose tissue. This paper shows the most recent results of large scale applications of density measurements and convinces us that the glandular content is much lower than generally assumed.

19. Wu X, Barnes GT, Tucker DM. Spectral dependence of glandular tissue dose in screen-film mammography. Radiology. 1991;179(1): $143-8$.
20. • Dance DR. Monte Carlo calculation of conversion factors for the estimation of mean glandular breast dose. Phys Med Biol. 1990;35(9):1211-9. Historical paper that was the basis for a lot of technical and scientific developments, mainly in the UK. Today the same approach is used, with new dose correction factors with new developments appearing on the market.

21. - Sechopoulos I, Bliznakova K, Qin X, et al. Characterization of the homogeneous tissue mixture approximation in breast imaging dosimetry. Med Phys. 2012;39(8):5050-9. New insights for improved breast dosimetry.

22. Sechopoulos I, Suryanarayanan S, Vedantham S, et al. Computation of the glandular radiation dose in digital tomosynthesis of the breast. Med Phys. 2007;34(1):221-32.

23. •• Dance DR, Young KC, van Engen RE. Estimation of mean glandular dose for breast tomosynthesis: factors for use with the UK, European and IAEA breast dosimetry protocols. Phys Med Biol. 2011;56(2):453-71. New paper that explains how to calculate the mean glandular dose for breast tomosynthesis.

24. Thacker SC, Glick SJ. Normalized glandular dose (DgN) coefficients for flat-panel CT breast imaging. Phys Med Biol. 2004;49(24):5433-44.

25. Dance DR, Skinner CL, Young KC, et al. Additional factors for the estimation of mean glandular breast dose using the UK mammography dosimetry protocol. Phys Med Biol. 2000;45(11): $3225-40$

26. Yaffe MJ. Mammographic density. Measurement of mammographic density. Breast Cancer Res. 2008;10(3):209. doi:10.1186/ bcr2102.Epub2008Jun19.

27. Kotre CJ. X-ray absorptiometry of the breast using mammographic exposure factors: application to units featuring automatic beam quality selection. Br J Radiol. 2010;83(990):515-23.

28. Geeraert N, Klausza R, Mullera S, et al. Breast characteristics and dosimetric data in X-ray mammography A large sample worldwide survey. Bonn: IAEA conference; 2012.

29. • Kotre CJ. Statistical analysis of mammographic breast composition measurements: towards a quantitative measure of relative breast cancer risk. Br J Radiol. 2011;84(998):153-60. This is a recent study to demonstrate a link between increased breast glandularity and increased risk for breast cancer. Similar studies could be performed prospectively with digital mammography using software tools to estimate the glandular volume.

30. Rosenberg RD, Kelsey CA, Williamson MR, et al. Computerbased collection of mammographic exposure data for quality assurance and dosimetry. Med Phys. 2001;28(8):1546-51.

31. • Chevalier M, Morán P, Ten JI, et al. Patient dose in digital mammography. Med Phys. 2004;31(9):2471-9. This paper was the first to show the opportunity offered by digital mammography to automate patient dose data collection.

32. Jacobs A, Cockmartin L, Dance DR, Bosmans H. Digital breast tomosynthesis: comparison of different methods to calculate patient doses. Vienna: IAEA conference IDOS; 2010.

33. •• Faulkner K, Law J, Robson KJ. Assessment of mean glandular dose in mammography. Br J Radiol. 1995;68(812):877-81. This paper estimates the total radiation detriment from $X$-ray imaging in screening and subsequent diagnostic imaging. The paper underlines the importance of focusing on dosimetry and dose optimisation of the screening part (as opposed to subsequent imaging).

34. Dance DR, Young KC, van Engen RE. Further factors for the estimation of mean glandular dose using the United Kingdom, European and IAEA breast dosimetry protocols. Phys Med Biol. 2009;54(14):4361-72. doi:10.1088/0031-9155/54/14/002.Epub 2009Jun23.

35. Dong SL, Chu TC, Lin YC, et al. Determination of equivalent breast phantoms for different age groups of Taiwanese women: an experimental approach. Med Phys. 2011;38(7):4094-100. 
36. Smans K, Bosmans H, Xiao M, et al. Towards a proposition of a diagnostic (dose) reference level for mammographic acquisitions in breast screening measurements in Belgium. Radiat Prot Dosimetry. 2005;117(1-3):321-6. Epub 2006 Feb 7.

37. Radiation protection 109. Guidance on diagnostic reference levels (DRLs) for medical exposures. European Commission, 1999.

38. - Tsapaki V, Tsalafoutas IA, Poga V, et al. Investigation of breast dose in five screening mammography centres in Greece. J Radiol Prot. 2008;28(3):337-46. Epub 2008 Aug 20. Document of the EC guiding the implementation of European Guidelines.

39. McCullagh JB, Baldelli P, Phelan N. Clinical dose performance of full field digital mammography in a breast screening programme. Br J Radiol. 2011;84(1007):1027-33. Epub 2011 May 17.

40. Gennaro G, Baldelli P, Taibi A, et al. Patient dose in full-field digital mammography: an Italian survey. Eur Radiol. 2004; 14(4):645-52. Epub 2003 Aug 12.

41. Vassileva J, Dimov A, Slavchev A, Karadjov A. Bulgarian experience in the establishment of reference dose levels and implementation of a quality control system in diagnostic radiology. Radiat Prot Dosimetry. 2005;117(1-3):131-4.

42. Verdun FR, Moeckli R, Valley JF, et al. Survey on image quality and dose levels used in Europe for mammography. Br J Radiol. 1996;69(824):762-8.

43. • Faulkner K, Wallis MG, Neilson F, Whitaker CJ. Evaluation of the population dose to the UK population from the National Health Service Breast Screening Programme. Radiat Prot Dosimetry. 2008;129(1-3):184-90. Epub 2008 May 14. This paper estimates the total radiation detriment from $X$-ray imaging in screening and subsequent diagnostic imaging. The paper underlines the importance of focusing on dosimetry and dose optimisation of the screening part (as opposed to subsequent imaging).

44. Young KC, Oduko JM, Bosmans H, et al. Optimal beam quality selection in digital mammography. Br J Radiol. 2006;79(948): 981-90.

45. Bernhardt P, Mertelmeier T, Hoheisel M. X-ray spectrum optimization of full-field digital mammography: simulation and phantom study. Med Phys. 2006;33(11):4337-49.

46. Toroi P, Zanca F, Young KC, et al. Experimental investigation on the choice of the tungsten/rhodium anode/filter combination for an amorphous selenium-based digital mammography system. Eur Radiol. 2007;17(9):2368-75. Epub 2007 Feb 1.
47. Ranger NT, Lo JY, Samei E. A technique optimization protocol and the potential for dose reduction in digital mammography. Med Phys. 2010;37(3):962-9.

48. • Perry N, Broeders M, de Wolf C, Törnberg S, Holland R, von Karsa L. European guidelines for breast cancer screening and diagnosis. Luxemburg: Office for the Official Publications of the European Communities; 2006. European protocol for Quality Assurance in breast cancer screening and diagnosis, widely used in Europe today.

49. IAEA Human Health Series, No 17. Quality assurance programme for digital mammography. http://www-pub.iaea.org/MTCD/ Publications/PDF/Pub1482_web.pdf.

50. Klausz R, Shramchenko N. Dose to population as a metric in the design of optimised exposure control in digital mammography. Radiat Prot Dosimetry. 2005;114(1-3):369-74.

51. Monnin P, Marshall NW, Bosmans H, et al. Image quality assessment in digital mammography: part II. NPWE as a validated alternative for contrast detail analysis. Phys Med Biol. 2011;56(14):4221-38.

52. Carton AK, Ullberg C, Maidment AD. Optimization of a dualenergy contrast-enhanced technique for a photon-counting digital breast tomosynthesis system: II. An experimental validation. Med Phys. 2010;37(11):5908-13.

53. McKinley RL, Tornai MP, Samei E, Bradshaw ML. Simulation study of a quasi-monochromatic beam for X-ray computed mammotomography. Med Phys. 2004 Apr, 31(4), pp. 800-13. Erratum in: Med Phys. 2004;31(11):3163.

54. Samei E, Saunders RS. Dual-energy contrast-enhanced breast tomosynthesis: optimization of beam quality for dose and image quality. Phys Med Biol. 2011;56(19):6359-78.

55. Segui JA, Zhao W. Amorphous selenium flat panel detectors for digital mammography: validation of a NPWE model observer with CDMAM observer performance experiments. Med Phys. 2006;33(10):3711-22.

56. Mainprize JG, Yaffe MJ. Cascaded analysis of signal and noise propagation through a heterogeneous breast model. Med Phys. 2010;37(10):5243-50.

57. Zanca F, Jacobs J, Van Ongeval C, et al. Evaluation of clinical image processing algorithms used in digital mammography. Med Phys. 2009;36(3):765-75. 